\title{
Multi-Objective Weighted Sampling
}

\author{
Edith Cohen \\ Google Research \\ Mountain View, CA, USA \\ edith@cohenwang.com
}

\begin{abstract}
Multi-objective samples are powerful and versatile summaries of large data sets. For a set of keys $x \in X$ and associated values $f_{x} \geq 0$, a weighted sample taken with respect to $f$ allows us to approximate segment-sum statistics $\operatorname{sum}(f ; H)=\sum_{x \in H} f_{x}$, for any subset $H$ of the keys, with statistically-guaranteed quality that depends on sample size and the relative weight of $H$. When estimating $\operatorname{sum}(g ; H)$ for $g \neq f$, however, quality guarantees are lost. A multi-objective sample with respect to a set of functions $F$ provides for each $f \in F$ the same statistical guarantees as a dedicated weighted sample while minimizing the summary size. We analyze properties of multi-objective samples and present sampling schemes and meta-algortithms for estimation and optimization while showcasing two important application domains. The first are key-value data sets, where different functions $f \in F$ applied to the values correspond to different statistics such as moments, thresholds, capping, and sum. A multi-objective sample allows us to approximate all statistics in $F$. The second is metric spaces, where keys are points, and each $f \in F$ is defined by a set of points $C$ with $f_{x}$ being the service cost of $x$ by $C$, and $\operatorname{sum}(f ; X)$ models centrality or clustering cost of $C$. A multi-objective sample allows us to estimate costs for each $f \in F$. In these domains, multi-objective samples are often of small size, are efficiently to construct, and enable scalable estimation and optimization. We aim here to facilitate further applications of this powerful technique.
\end{abstract}

\section{INTRODUCTION}

Random sampling is a powerful tool for working with very large data sets on which exact computation, even of simple statistics, can be time and resource consuming. A small sample of the data 2llows us to efficiently obtain approximate answers.

in Consider data in the form of key value pairs $\left\{\left(x, f_{x}\right)\right\}$, where Veys $x$ are from some universe $\mathcal{X}, f_{x} \geq 0$, and we define $f_{x} \equiv 0$ for keys $x \in \mathcal{X}$ that are not present in the data. Very common statistics over such data are segment sum statistics

$$
\operatorname{sum}(f ; H)=\sum_{x \in H} f_{x},
$$

where $H \subset \mathcal{X}$ is a segmen $\mathbb{1}^{1}$ of $\mathcal{X}$. Examples of such data isets are IP flow keys and bytes, users and activity, or customers - and distance to the nearest facility. Segments may correspond to 3 certain demographic or location or other meta data of keys. Segment statistics in these example correspond respectively to total traffic, activity, or service cost of the segment.

When the data set is large, we can compute a weighted sample which includes each key $x$ with probability (roughly) proportional to $f_{x}$ and allows us to estimate segment sum statistics $\widehat{\text { sum }}(f ; H)$ for query segments $H$. Popular weighted sampling schemes [30], [32] include Poisson Probability Proportional to Size (pps) [21], VarOpt [5], [12], and the bottom- $k$ schemes [28], [14], [15] Sequential Poisson (priority) [24], [17] and PPS without replacement (ppswor) [27].

These weighted samples provide us with nonnegative unbiased estimates $\widehat{\operatorname{sum}}(g ; H)$ for any segment and $g \geq 0$ (provided that $f_{x}>0$ when $\left.g_{x}>0\right)$. For all statistics $\operatorname{sum}(f ; H)$ we obtain statistical guarantees on estimation quality: The error, measured by the coefficient of variation (CV), which is the standard deviation divided by the mean, is at most the inverse

1. The alternative term selection is used in the DB literature and the term domain is used in the statistics literature. of the square root of the size of the sample multiplied by the fraction $\operatorname{sum}(f ; H) / \operatorname{sum}(f, \mathcal{X})$ of " weight" that is due to the segment $H$. This trade-off of quality (across segments) and sample size are (worst-case) optimal. Moreover, the estimates are wellconcentrated in the Chernoff-Bernstein sense: The probability of an error that is $c$ times the $\mathrm{CV}$ decreases exponentially in $c$.

In many applications, such as the following examples, there are multiple sets of values $f \in F$ that are associated with the keys: (i) Data records can come with explicit multiple weights, as with activity summaries of customers/jobs that specify both bandwidth and computation consumption. (ii) Metric objectives, such as our service cost example, where each configuration of facility locations induces a different set of distances and hence service costs. (iii) The raw data can be specified in terms of a set of key value pairs $\left\{\left(x, w_{x}\right)\right\}$ but we are interested in different functions $f_{x} \equiv f\left(w_{x}\right)$ of the values that correspond to different statistics such as

\begin{tabular}{l|l} 
Statistics & function $f(w)$ \\
\hline count & $f(w)=1$ for $w>0$ \\
sum & $f(w)=w$ \\
threshold with $T>0$ & thresh $_{T}(w)=I_{w \geq T}$ \\
moment with $p>0$ & $f(w)=w^{p}$ \\
capping with $T>0$ & $\operatorname{cap}_{T}=\min \{T, w\}$
\end{tabular}

Example 1.1. Consider a toy data set $\mathcal{D}$ :

$(u 1,5),(u 3,100), \quad(u 10,23),(u 12,7),(u 17,1), \quad(u 24,5)$, (u31, 220), (u42, 19), (u43,3), (u55,2)

For a segment $H$ with $H \cap \mathcal{D}=\{u 3, u 12, u 42, u 55\}$, we have $\operatorname{sum}(H)=128$, $\operatorname{count}(H)=4$, thresh $h_{10}(H)=2$, $\operatorname{cap}_{5}(H)=17$, and 2-moment $(H)=10414$.

For these applications, we are interested in a summary that can provide us with estimates with statistically guaranteed quality for each $f \in F$. The naive solutions are not satisfactory: We can compute a weighted sample taken with respect to a particular $f \in F$, but the quality of the estimates $\operatorname{sum}(g ; H)$ rapidly 
degrades with the dissimilarity between $g$ and $f$. We can compute a dedicated sample for each $f \in F$, but the total summary size can be much larger than necessary. Multi-objective samples, a notion crystallized in $[16]^{2}$, provide us with the desired statistical guarantees on quality with minimal summary size.

Multi-objective samples build on the classic notion of sample coordination [23], [4], [29], [7], [28], [25]. In a nutshell, coordinated samples are locality sensitive hashes of $f$, mapping similar $f$ to similar samples. A multi-objective sample is (roughly) the union $S^{(F)}=\bigcup_{f \in F} S^{(f)}$ of all the keys that are included in coordinated weighted samples $S^{(f)}$ for $f \in F$. Because the samples are coordinated, the number of distinct keys included and hence the size of $S^{(F)}$ is (roughly) as small as possible. Since for each $f \in F$, the sample $S^{(F)}$ "includes" the dedicated sample $S^{(f)}$, the estimate quality from $S^{(F)}$ dominates that of $S^{(f)}$.

In this paper, we review the definition of multi-objective samples, study their properties, and present efficient sampling schemes. We consider both general sets $F$ of objectives and families $F$ with special structure. By exploiting special structure, we can bound the overhead, which is the increase factor in sample size necessary to meet multi-objective quality guarantees, and obtain efficient sampling schemes that avoid dependence of the computation on $|F|$.

\subsection{Organization}

In Section 2 we review (single-objective) weighted sampling focusing on the Poisson pps and bottom- $k$ sampling schemes. We then review the definitions [16] and establish properties of multi-objective samples. In Section 3 we study multi-objective pps samples. We show that the multi-objective sample size is also necessary for meeting the quality guarantees for segment statistics for all $f \in F$. We also show that the guarantees are met when we use upper bounds on the multi-objective pps sampling probabilities instead of working with the exact values. In Section 4 we study multi-objective bottom- $k$ samples.

In Section 5 we establish a fundamental property of multiobjective samples: We define the sampling closure $\bar{F}$ of a set of objectives $F$, as all functions $f$ for which a multi-objective sample $S^{(F)}$ meets the quality guarantees for segment statistics. Clearly $F \subset \bar{F}$ but we show that the closure $\bar{F}$ also includes every $f$ that is a non-negative linear combination of functions from $F$.

In Section 6, we consider data sets in the form of key value pairs and the family $M$ of all monotone non-decreasing functions of the values. This family includes most natural statistics, such as our examples of count, sum, threshold, moments, and capping. Since $M$ is infinite, it is inefficient to apply a generic multi-objective sampling algorithm to compute $S^{(M)}$. We present efficient near-linear sampling schemes for $S^{(M)}$ which also apply over streamed or distributed data. Moreover, we establish a bound on the sample size of $\mathrm{E}\left[\left|S^{(M)}\right|\right] \leq k \ln n$, where $n$ is the number of keys in our data set and $k$ is the reference size of the singleobjective samples $S^{(f)}$ for each $f \in M$. The design is based on a surprising relation to All-Distances Sketches [7], [8]. Furthermore, we establish that (when key weights are unique), a sample of size $\Omega(k \ln n)$ is necessary: Intuitively, the "hardness" stems from the need to support all threshold functions.

In Section 7 we study the set $C=\left\{\operatorname{cap}_{T} \mid T>0\right\}$ of all capping functions. The closure $\bar{C}$ includes all concave $f \in M$

2. The collocated model with at most a linear growth (satisfy $f^{\prime}(x) \leq 1$ and $f^{\prime \prime}(x) \leq 0$ ). Since $C \subset M$, the multi-objective sample $S^{(M)}$ includes $S^{(C)}$ and provides estimates with statistical guarantees for all $f \in \bar{C}$. The more specialized sample $S^{(C)}$, however, can be much smaller than $S^{(M)}$. We design an efficient algorithm for computing $S^{(C)}$ samples.

In Section 8 we discuss metric objectives and multi-objective samples as summaries of a set of points that allows us to approximate such objectives.

In Section 9 we discuss different types of statistical guarantees across functions $f$ in settings where we are only interested in statistics $\operatorname{sum}(f ; \mathcal{X})$ over the full data. Our basic multi-objective samples analyzes the sample size required for ForEach, where the statistical guarantees apply to each estimate $\widehat{\operatorname{sum}}(f ; H)$ in isolation. In particular, also to each estimate over the full data set. ForAll is much stronger and bounds the (distribution of) the maximum relative error of estimates $\widehat{\operatorname{sum}}(f ; \mathcal{X})$ for all $f \in F$. Meeting ForAll typically necessitates a larger multi-objective sample size than meeting ForEach.

In section 10 we present a meta-algorithm for optimization over samples. The goal is to maximize a (smooth) function of $\operatorname{sum}(f ; \mathcal{X})$ over $f \in F$. When $\mathcal{X}$ is large, we can instead perform the optimization over a small multi-objective sample of $\mathcal{X}$. This framework has important applications to metric objectives and estimating loss of a model from examples. The ForOpt guarantee is for a sample size that facilitates such optimization, that is, the approximate maximizer over the sample is an approximate maximizer over the data set. This guarantee is stronger than ForEach but generally weaker than ForAll. We make a key observation that with a ForEach sample we are only prone to testable onesided errors on the optimization result. Based on that, we present an adaptive algorithm where the sample size is increased until ForOpt is met. This framework unifies and generalizes previous work of optimization over coordinated samples [13], [11].

We conclude in Section 11

\section{Weighted sampling (SINGLe obJeCtive)}

We review weighted sampling schemes with respect to a set of values $f_{x}$, focusing on preparation for the multi-objective generalization. The schemes are specified in terms of a samplesize parameter $k$ which allows us to trade-off representation size and estimation quality.

\subsection{Poisson Probability Proportional to Size (pps)}

The pps sample $S^{(f, k)}$ includes each key $x$ independently with probability

$$
p_{x}^{(f, k)}=\min \left\{1, k \frac{f_{x}}{\sum_{y} f_{y}}\right\} .
$$

Example 2.1. The table below lists pps sampling probabilities $p_{x}^{(f, 3)}(k=3$, rounded to the nearest hundredth) for keys in our example data for $\operatorname{sum}\left(f_{x}=w_{x}\right)$, thresh $h_{10}\left(f_{x}=I_{w_{x} \geq 10}\right)$, and $\operatorname{cap}_{5}\left(f_{x}=\min \left\{5, w_{x}\right\}\right)$. The number in parenthesis is $\operatorname{sum}(f, \mathcal{X})=\sum_{x} f_{x}$. We can see that sampling probabilities highly vary between functions $f$.

\begin{tabular}{l||r|r|r|r|r|r|r|r|r|r}
\hline key & $u I$ & $u 3$ & $u I 0$ & $u I 2$ & $u I 7$ & $u 24$ & $u 31$ & $u 42$ & $u 43$ & $u 55$ \\
$w_{x}$ & 5 & 100 & 23 & 7 & 1 & 5 & 220 & 19 & 3 & 2 \\
\hline sum (385) $_{\text {thresh }}(4)$ & 0.04 & 0.78 & 0.18 & 0.05 & 0.01 & 0.04 & 1.00 & 0.15 & 0.02 & 0.02 \\
cap $_{5}(41)$ & 0.00 & 0.75 & 0.75 & 0.00 & 0.00 & 0.00 & 0.75 & 0.75 & 0.00 & 0.00 \\
& 0.37 & 0.37 & 0.37 & 0.37 & 0.07 & 0.37 & 0.37 & 0.37 & 0.22 & 0.15
\end{tabular}

PPS samples can be computed by association a random value $u_{x} \sim U[0,1]$ with each key $x$ and including the key in the sample 
if $u_{x} \leq p_{x}^{(f, k)}$. This formulation to us when there are multiple objectives as it facilitates the coordination of samples taken with respect to the different objectives. Coordination is achieved using the same set $u_{x}$.

\subsection{Bottom- $k$ (order) sampling}

Bottom- $k$ sampling unifies priority (sequential Poisson) [24], [17] and pps without replacement (ppswor) sampling [27]. To obtain a bottom- $k$ sample for $f$ we associate a random value $u_{x} \sim U[0,1]$ with each key. To obtain a ppswor sample we use $r_{x} \equiv-\ln (1-$ $\left.u_{x}\right)$ and to obtain a priority sample we use $r_{x} \equiv u_{x}$. The bottom$k$ sample $S^{(f, k)}$ for $f$ contains the $k$ keys with minimum $f$-seed, where

$$
f \text {-seed }(x) \equiv \frac{r_{x}}{f_{x}} .
$$

To support estimation, we also retain the threshold, $\tau^{(f, k)}$, which is defined to be the $(k+1)$ st smallest $f$-seed.

\subsection{Estimators}

We estimate a statistics $\operatorname{sum}(g ; H)$ from a weighted sample $S^{(f, k)}$ using the inverse probability estimator [22]:

$$
\widehat{\operatorname{sum}}(g ; H)=\sum_{x \in H \cap S} \frac{g_{x}}{p_{x}^{(f, k)}} .
$$

The estimate is always nonnegative and is unbiased when the functions satisfy $g_{x}>0 \Longrightarrow f_{x}>0$ (which ensures that any key $x$ with $g_{x}>0$ is sampled with positive probability). To apply this estimator, we need to compute $p_{x}^{(f, k)}$ for $x \in S$. To do so with pps samples (1) we include the sum $\sum_{x} f_{x}$ with $S$ as auxiliary information.

For bottom- $k$ samples, inclusion probabilities of keys are not readily available. We therefore use the inverse probability estimator (2) with conditional probabilities $p_{x}^{(f, k)}$ [17], [15]: A key $x$, fixing the randomization $u_{y}$ for all other keys, is sampled if and only if $f$-seed $(x)<t$, where $t$ is the $k$ th smallest $f$ seed among keys $y \neq x$. For $x \in S^{(f, k)}$, the $k$ th smallest $f$-seed among other keys is $t=\tau^{(f, k)}$, and thus

$$
p_{x}^{(f, k)}=\operatorname{Pr}_{u_{x} \sim U[0,1]}\left[\frac{r_{x}}{f_{x}}<\tau^{(f, k)}\right] .
$$

Note that the right hand side expression for probability is equal to $1-e^{-f_{x} t}$ with ppswor and to $\min \left\{1, f_{x} t\right\}$ with priority sampling.

\subsection{Estimation quality}

We consider the variance and concentration of our estimates. A natural measure of estimation quality of our unbiased estimates is the coefficient of variation (CV), which is the ratio of the standard deviation to the mean. We can upper bound the $\mathrm{CV}$ of our estimates 2 of $\operatorname{sum}(g ; H)$ in terms of the (expected) sample size $k$ and the relative $g$-weight of the segment $H$, defined as

$$
q^{(g)}(H)=\frac{\operatorname{sum}(g ; H)}{\operatorname{sum}(g ; \mathcal{X})} .
$$

To be able to express a bound on the $\mathrm{CV}$ when we estimate a statistics $\operatorname{sum}(g ; H)$ using a weighted sample taken with respect to $f$, we define the disparity between $f$ and $g$ as

$$
\rho(f, g)=\max _{x} \frac{f_{x}}{g_{x}} \max _{x} \frac{g_{x}}{f_{x}} .
$$

The disparity always satisfies $\rho(f, g) \geq 1$ and we have equality $\rho(f, g)=1$ only when $g$ is a scaling of $f$, that is, equal to $g=c f$ for some $c>0$. We obtain the following upper bound:

Theorem 2.1. For pps samples and the estimator (2),

$$
\forall g \forall H, C V[\widehat{\operatorname{sum}}(g ; H)] \leq \sqrt{\frac{\rho(f, g)}{q^{(g)}(H) k}} .
$$

For bottom- $k$ samples, we replace $k$ by $k-1$.

The proof for $\rho=1$ is standard for pps, provided in [7], [8] for ppswor, and in [31] for priority samples. The proof for $\rho \geq 1$ for ppswor is provided in Theorem A.1. The proof for pps is simpler, using a subset of the arguments. The proof for priority can be obtained by generalizing [31].

Moreover, the estimates obtained from these weighted sample are concentrated in the Chernoff-Hoeffding-Bernstein sense. We provide the proof for the multiplicative form of the bound and for Poisson pps samples:

Theorem 2.2. For $\delta \leq 1$,

$$
\begin{aligned}
& \operatorname{Pr}[|\widehat{\operatorname{sum}}(g ; H)-\operatorname{sum}(g ; H)|>\delta \operatorname{sum}(g ; H)] \\
& \quad \leq 2 \exp \left(-q^{(g)}(H) k \rho^{-2} \delta^{2} / 3\right) .
\end{aligned}
$$

For $\delta>1$,

$$
\begin{aligned}
& \operatorname{Pr}[\widehat{\operatorname{sum}}(g ; H)-\operatorname{sum}(g ; H)>\delta \operatorname{sum}(g ; H)] \\
& \quad \leq \exp \left(-q^{(g)}(H) k \rho^{-2} \delta / 3\right) .
\end{aligned}
$$

Proof. Consider Poisson pps sampling and the inverse probability estimator. The contribution of keys that are sampled with $p_{x}^{(f, k)}=$ 1 is computed exactly. Let the contribution of these keys be ( $1-$ $\alpha) \operatorname{sum}(g ; H)$, for some $\alpha \in[0,1])$. If $\alpha=0$, the estimate is the exact sum and we are done. Otherwise, it suffices to estimate the remaining $\alpha \operatorname{sum}(g ; H)$ with relative error $\delta^{\prime}=\delta / \alpha$.

Consider the remaining keys, which have inclusion probabilities $p_{x}^{(f, k)} \geq k f_{x} / \operatorname{sum}(f)$. The contribution of such a key $x$ to the estimate is 0 if $x$ is not sampled and is

$$
\frac{g_{x}}{p_{x}^{(f, k)}} \leq \rho(f, g) \operatorname{sum}(f) / k
$$

when $x$ is sampled. Note that by definition

$$
\operatorname{sum}(g ; H)=q^{(g)}(H) \operatorname{sum}(g) \geq q^{(g)}(H) \operatorname{sum}(f) / \rho(f, g) \text {. }
$$

We apply the concentration bounds to the sum of random variables in the range $[0, \rho(f, g) \operatorname{sum}(f) / k]$. To use the standard form, we can normalize our random variables and to have range $[0,1]$ and accordingly normalize the expectation $\alpha \operatorname{sum}(g ; H)$ to obtain

$$
\mu=\alpha \frac{\operatorname{sum}(g ; H)}{\rho(f, g) \operatorname{sum}(f) / k]} \geq \alpha q^{(g)}(H) \rho(f, g)^{-2} k
$$

We can now apply multiplicative Chernoff bounds for random variables in the range $[0,1]$ with $\delta^{\prime}=\delta / \alpha$ and expectation $\mu$. The formula bounds the probability of relative error that exceeds $\delta$ by $2 \exp \left(-\delta^{2} \mu / 3\right.$ when $\delta<1$ and by $\exp (-\delta \mu / 3$ when $\delta>1$.

\subsection{Computing the sample}

Consider data presented as streamed or distributed elements of the form of key-value pairs $\left(x, f_{x}\right)$, where $x \in \mathcal{X}$ and $f_{x}>0$. We define $f_{x} \equiv 0$ for keys $x$ that are not in the data.

An important property of our samples (bottom- $k$ or pps) is that they are composable (mergeable). Meaning that a sample of 
the union of two data sets can be computed from the samples of the data sets. Composability facilitates efficient streamed or distributed computation. The sampling algorithms can use a random hash function applied to key $x$ to generate $u_{x}$ - so seed values can be computed on the fly from $\left(x, f_{x}\right)$ and do not need to be stored.

With bottom- $k$ sampling we permit keys $x$ to occur in multiple elements, in which case we define $f_{x}$ to be the maximum value of elements with key $x$. The sample $S(\mathcal{D})$ of a set $\mathcal{D}$ of elements contains the pair $\left(x, f_{x}\right)$ for the $k+1$ (unique) keys with smallest $f$-seeds ${ }^{3}$ The sample of the union $\bigcup_{i} \mathcal{D}_{i}$ is obtained from $\bigcup_{i} S\left(\mathcal{D}_{i}\right)$ by first replacing multiple occurrences of a key with the one with largest $f(w)$ and then returning the pairs for the $k+1$ keys with smallest $f$-seeds.

With pps sampling, the information we store with our sample $S(\mathcal{D})$ includes the sum $\operatorname{sum}(f ; \mathcal{D}) \equiv \sum_{x \in D} f_{x}$ and the sampled pairs $\left(x, f_{x}\right)$, which are those with $u_{x} \leq k f_{x} / \operatorname{sum}(f ; \mathcal{D})$. Because we need to accurately track the sum, we require that elements have unique keys. The sample of a union $D=\bigcup_{i} \mathcal{D}_{i}$ is obtained using the $\operatorname{sum} \operatorname{sum}(f ; \mathcal{D})=\sum_{i} \operatorname{sum}\left(f ; \mathcal{D}_{i}\right)$, and retaining only keys in $\bigcup_{i} S\left(\mathcal{D}_{i}\right)$ that satisfy $u_{x} \leq k f_{x} / \operatorname{sum}(f ; \mathcal{D})$.

\section{Multi-OBJective pPS SAMPLES}

Our objectives are specified as pairs $\left(f, k_{f}\right)$ where $f \in F$ is a function and $k_{f}$ specifies a desired estimation quality for $\operatorname{sum}(f ; H)$ statistics, stated in terms of the quality (Theorem 2.1 and Theorem 2.2 provided by a single-objective sample for $f$ with size parameter $k_{f}$. To simplify notation, we sometimes omit $k_{f}$ when clear from context.

A multi-objective sample $S^{(F)}$ [16] is defined by considering dedicated samples $S^{\left(f, k_{f}\right)}$ for each objective that are coordinated. The dedicated samples are coordinating by using the same randomization, which is the association of $u_{x} \sim U[0,1]$ with keys. The multi-objective sample $S^{(F)}=\bigcup_{f \in F} S^{\left(f, k_{f}\right)}$ contains all keys that are included in at least one of the coordinated dedicated samples. In the remaining part of this section we study pps samples. Multi-objective bottom- $k$ samples are studied in the next section.

Lemma 3.1. A multi-objective pps sample for $F$ includes each key $x$ independently with probability

$$
p_{x}^{(F)}=\min \left\{1, \max _{f \in F} \frac{k_{f} f_{x}}{\sum_{y} f_{y}}\right\} .
$$

Proof. Consider coordinated dedicated pps samples for $f \in F$ obtained using the same set $\left\{u_{x}\right\}$. The key $x$ is included in at least one of the samples if and only if the value $u_{x}$ is at most the maximum over objectives $\left(f, k_{f}\right)$ of the pps inclusion probability for that objective:

$$
\begin{aligned}
u_{x} & \leq \max _{f \in F} p_{x}^{\left(f, k_{f}\right)}=\max _{f \in F} \min \left\{1, \frac{k_{f} f_{x}}{\sum_{y} f_{y}}\right\} \\
& =\min \left\{1, \max _{f \in F} \frac{k_{f} f_{x}}{\sum_{y} f_{y}}\right\} .
\end{aligned}
$$

Since $u_{x}$ are independent, so are the inclusion probabilities of different keys.

Example 3.2. Consider the three objectives: sum, thresh $h_{10}$, and cap $_{5}$ all with $k=3$ as in Example 2.1. The expected size of $S^{(F)}$

3. When keys are unique to elements it suffices to keep only the $(k+1)$ st smallest $f$-seed without the pair $\left(x, f_{x}\right)$. is $\left|S^{(F)}\right|=\sum_{x} p_{x}^{(F)}=4$ 4.68. The naive solution of maintaining a separate dedicated sample for each objective would have total expected size 8.29 (Note that the dedicated expected sample size for sum is 2.29 and for thresh ${ }_{10}$, and cap $_{5}$ it is 3 .

To estimate a statistics $\operatorname{sum}(g ; H)$ from $S^{(F)}$, we apply the inverse probability estimator

$$
\widehat{\operatorname{sum}}(g ; H)=\sum_{x \in S^{(F)} \cap H} \frac{g_{x}}{p_{x}^{(F)}} .
$$

using the probabilities $p_{x}^{(F)}$ 4.

To compute the estimator (5), we need to know $p_{x}^{(F)}$ when $x \in S^{(F)}$. These probabilities can be computed if we maintain the sums $\operatorname{sum}(f)=\sum_{x} f_{x}$ for $f \in F$ as auxiliary information and we have $f_{x}$ available to us when $x \in S^{\left(f, k_{f}\right)}$.

In some settings it is easier to obtain upper bounds $\pi_{x} \geq p_{x}^{(F)}$ on the multi-objective pps inclusion probabilities, compute a Poisson sample using $\pi_{x}$, and apply the respective inverse-probability estimator

$$
\widehat{\operatorname{sum}}(g ; H)=\sum_{x \in S \cap H} \frac{g_{x}}{\pi_{x}} .
$$

Side note: It is sometime useful to use other sampling schemes, in particular, VarOpt (dependent) sampling [5], [20], [12] to obtain a fixed sample size. The estimation quality bounds on the CV and concentration also hold with VarOpt (which has negative covariances).

\subsection{Multi-objective pps estimation quality}

We show that the estimation quality, in terms of the bounds on the CV and concentration, of the estimator (5) is at least as good as that of the estimate we obtain from the dedicated samples. To do so we prove a more general claim that holds for any Poisson sampling scheme that includes each key $x$ in the sample $S$ with probability $\pi_{x} \geq p^{\left(f, k_{f}\right)}$ and the respective inverse probability estimator (6).

The following lemma shows that estimate quality can only improve when inclusion probabilities increase:

Lemma 3.3. The variance var $[\widehat{\operatorname{sum}}(g ; H)]$ of (6) and hence $C V[\widehat{\operatorname{sum}}(g ; H)]$ are non-increasing in $\pi_{x}$.

Proof. For each key $x$ consider the inverse probability estimator $\hat{g}_{x}=g_{x} / \pi_{x}$ when $x$ is sampled and $\hat{g}_{x}=0$ otherwise. Note that $\operatorname{var}\left[\hat{g}_{x}\right]=g_{x}^{2}\left(1 / \pi_{x}-1\right)$, which is decreasing with $\pi_{x}$. We have $\widehat{\operatorname{sum}}(g ; H)]=\sum_{x \in H} \hat{g}_{x}$. When covariances between $\hat{g}_{x}$ are nonpositive, which is the case in particular with independet inclusions, we have $\operatorname{var}[\widehat{\operatorname{sum}}(g ; H)]=\sum_{x \in H} \operatorname{var}\left[\hat{g}_{x}\right]$ and the claim follows as it applies to each summand.

We next consider concentration of the estimates.

Lemma 3.4. The concentration claim of Theorem 2.2 carries over when we use the inverse probability estimator with any sampling probabilities that satisfy $\pi_{x} \geq p_{x}^{(f, k)}$ for all $x$.

Proof. The generalization of the proof is immediate, as the range of the random variables $\hat{f}_{x}$ can only decrease when we increase the inclusion probability. 


\subsection{Uniform guarantees across objectives}

An important special case is when $k_{f}=k$ for all $f \in F$, that is, we seek uniform statistical guarantees for all our objectives. We use the notation $S^{(F, k)}$ for the respective multi-objective sample. We can write the multi-objective pps probabilities (4) as

$$
p_{x}^{(F, k)}=\min \left\{1, k \max _{f \in F} \frac{f_{x}}{\sum_{y} f_{y}}\right\}=\min \left\{1, k p_{x}^{(F, 1)}\right\} .
$$

The last equality follows when recalling the definitions of $p_{x}^{(f, 1)}=$ $f_{x} / \sum_{y} f_{y}$ and hence

$$
p_{x}^{(F, 1)}=\max _{f \in F} p_{x}^{(f, 1)}=\max _{f \in F} \frac{f_{x}}{\sum_{y} f_{y}} .
$$

We refer to $p_{x}^{(f, 1)}$ as the base pps probabilities for $f$. Note that the base pps probabilities are rescaling of $f$ and pps probabilities are invariant to this scaling. We refer to $p_{x}^{(F, 1)}$ as the multiobjective base pps probabilities for $F$. Finally, for a reason that will soon be clear, we refer to the sum

$$
h^{(\mathrm{pps})}(F) \equiv \sum_{x} p_{x}^{(F, 1)}
$$

as the multi-objective pps overhead of $F$.

It is easy to see that $h(F) \in[1,|F|]$ and $h(F)$ is closer to 1 when all objectives in $F$ are more similar. We can write

$$
k p_{x}^{(F, 1)}=(k h(F)) \frac{p_{x}^{(F, 1)}}{\sum_{x} p_{x}^{(F, 1)}} .
$$

That is, the multi-objective pps probabilities (7) are equivalent to single-objective pps probabilities with size parameter $k h(F)$ computed with respect to base probability "weights" $g_{x}=p_{x}^{(F, 1)}$.

Side note: We can apply any single-objective weighted sampling scheme such as VarOpt or bottom- $k$ to the weights $p_{x}^{(F, 1)}$, or to upper-bounds $\pi_{x} \geq p_{x}^{(F, 1)}$ on these weights while adjusting the sample size parameter to $k \sum_{x} \pi_{x}$.

\subsection{Lower bound on multi-objective sample size}

The following theorem shows that any multi-objective sample for $F$ that meets the quality guarantees on all domain queries for $\left(f, k_{f}\right)$ must include each key $x$ with probability at least $\frac{p_{x}^{(f, 1)} k_{f}}{p_{x}^{(f, 1)} k_{f}+1} \geq \frac{1}{2} p_{x}^{\left(f, k_{f}\right)}$. Moreover, when $p_{x}^{(f, 1)} k_{f} \ll 1$, then the lower bound on inclusion probability is close to $p_{x}^{\left(f, k_{f}\right)}$. This implies the multi-objective pps sample size is necessary to meet the quality guarantees we seek (Theorem 2.1).

Theorem 3.1. Consider a sampling scheme that for weights $f$ supports estimators that satisfy for each segment $H$

$$
C V[\widehat{\operatorname{sum}}(f ; H)] \leq 1 / \sqrt{q^{(f)}(H) k} .
$$

Then the inclusion probability of a key $x$ must be at least

$$
p_{x} \geq \frac{p^{(f, 1)} k}{p^{(f, 1)} k+1} \geq \frac{1}{2} \min \left\{1, p^{(f, 1)} k\right\} .
$$

Proof. Consider a segment of a single key $H=\{x\}$. Then $p^{(f, 1)} \equiv q^{(f)}(H)=f_{x} / \sum_{y} f_{y} \equiv q$. The best nonnegative unbiased sum estimator is the HT estimator: When the key is not sampled, there is no evidence of the segment and the estimate must be 0 . When it is, uniform estimate minimize the variance. If the key is included with probability $p$, the $\mathrm{CV}$ of the estimate is

$$
\mathrm{C} V[\widehat{\operatorname{sum}}(f ;\{x\})]=(1 / p-1)^{0.5} .
$$

From the requirement $(1 / p-1)^{0.5} \leq 1 /(q k)^{0.5}$, we obtain that $p \geq \frac{q k}{q k+1}$.

\section{Multi-objective вотtom- $k$ SAmples}

The sample $S^{(F)}$ is defined with respect to random $\left\{u_{x}\right\}$. Each dedicated sample $S^{\left(f, k_{f}\right)}$ includes the $k_{f}$ lowest $f$-seeds, computed using $\left\{u_{x}\right\} . S^{(F)}$ accordingly includes all keys that have one of the $k_{f}$ lowest $f$-seeds for at least one $f \in F$.

To estimate statistics $\operatorname{sum}(g ; H)$ from bottom- $k S^{(F)}$, we again apply the inverse probability estimator $(5)$ but here we use the conditional inclusion probability $p_{x}^{(F)}$ for each key $x$ [16]. This is the probability (over $u_{x} \sim U[0,1]$ ) that $x \in S^{(F)}$, when fixing $u_{y}$ for all $y \neq x$ to be as in the current sample. Note that

$$
p_{x}^{(F)}=\max _{f \in F} p_{x}^{(f)},
$$

where $p_{x}^{(f)}$ are as defined in (3).

In order to compute the probabilities $p_{x}^{(F)}$ for $x \in$ $S^{(F)}$, it always suffices to maintain the slightly larger sample $\bigcup_{f \in F} S^{\left(f, k_{f}+1\right)}$. For completeness, we show that it suffices to instead maintain with $S^{(F)} \equiv \bigcup_{f \in F} S^{\left(f, k_{f}\right)}$ a smaller (possibly empty) set $Z \subset \bigcup_{f \in F} S^{\left(f, k_{f}+1\right)} \backslash S^{(F)}$ of auxiliary keys. We now define the set $Z$ and show how inclusion probabilities can be computed from $S^{(F)} \cup Z$. For a key $x \in S^{(F)}$, we denote by

$$
g^{(x)}=\arg \max _{f \in F \mid x \in S^{(f)}} p_{x}^{(f)}
$$

the objective with the most forgiving threshold for $x$. If $p_{x}^{\left(g^{(x)}\right)}<$ 1 , let $y_{x}$ be the key with $(k+1)$ smallest $g$-seed (otherwise $y_{x}$ is not defined). The auxiliary keys are then $Z=\left\{y_{x} \mid x \in S^{\left(F^{F}\right)}\right\} \backslash$ $S^{(F)}$. We use the sample and auxiliary keys $S^{(F)} \cup Z$ as follows to compute the inclusion probabilities: We first compute for each $f \in$ $F$, $\tau_{f}^{\prime}$, which is the $k_{f}+1$ smallest $f$-seed of keys in $S^{(F)} \cup Z$. For each $x \in S^{(F)}$, we then use $p_{x}^{(F)}=\max _{f \in F} f\left(w_{x}\right) \tau_{f}^{\prime}$ (for priority) or $p_{x}^{(F)}=1-\exp \left(-\max _{f \in F} f\left(w_{x}\right) \tau_{f}^{\prime}\right)$ (for ppswor). To see that $p_{x}^{(F)}$ are correctly computed, note that while we can have $\tau_{f}^{\prime}>\tau^{\left(f, k_{f}\right)}$ for some $f \in F(Z$ may not include the threshold keys of all the dedicated samples $S^{\left(f, k_{f}\right)}$ ), our definition of $Z$ ensures that $\tau_{f}^{\prime}=\tau^{\left(f, k_{f}\right)}$ for $f$ such that there is at least one $x$ where $f=g^{(x)}$ and $p_{x}^{\left(g^{(x)}\right)}<1$.

Composability: Note that multi-objective samples $S^{(F)}$ are composable, since they are a union of (composable) single-objective samples $S^{(f)}$. It is not hard to see that composability applies with the auxiliary keys: The set of auxiliary keys in the composed sample must be a subset of sampled and auxiliary keys in the components. Therefore, the sample itself includes all the necessary state for streaming or distributed computation.

Estimate quality: We can verify that for any $f \in F$ and $x$, for any random assignment $\left\{u_{y}\right\}$ for $y \neq x$, we have $p_{x}^{(F)} \geq p_{x}^{(f)}$. Therefore (applying Lemma 3.3 and noting zero covariances[15]) the variance and the CV are at most that of the estimator (2) applied to the bottom- $k_{f}$ sample $S^{(f)}$. To summarize, we obtain the following statistical guarantees on estimate quality with multiobjective samples: 
Theorem 4.1. For each $H$ and $g$, the inverse-probability estimator applied to a multi-objective pps sample $S^{(F)}$ has

$$
C V[\widehat{\operatorname{sum}}(g ; H)] \leq \min _{f \in F} \sqrt{\frac{\rho(f, g)}{q^{(g)}(H) k_{f}}} .
$$

The estimator applied to a multi-objective bottom- $k$ samples has the same guarantee but with $\left(k_{f}-1\right)$ replacing $k_{f}$.

Sample size overhead: We must have $\mathrm{E}\left[\left|S^{(F)}\right|\right] \leq \sum_{f \in F} k_{f}$. The worst-case, where the size of $S^{(F)}$ is the sum of the sizes of the dedicated samples, materializes when functions $f \in F$ have disjoint supports. The sample size, however, can be much smaller when functions are more related.

With uniform guarantees $\left(k_{f} \equiv k\right)$, we define the multiobjective bottom- $k$ overhead to be

$$
h^{(\text {botk })}(F) \equiv \mathrm{E}\left[\left|S^{(F)}\right|\right] / k .
$$

This is the sample size overhead of a multi-objective versus a dedicated bottom- $k$ sample.

pps versus bottom- $k$ multi-objective sample size: For some sets $F$, with the same parameter $k$, we can have much larger multiobjective overhead with bottom- $k$ than with pps. A multi-objective pps samples is the smallest sample that can include a pps sample for each $f$. A multi-objective bottom- $k$ sample must include a bottom- $k_{f}$ sample for each $f$. Consider a set of $n>k$ keys. For each subset of $n / 2$ keys we define a function $f$ that is uniform on the subset and 0 elsewhere. It is easy to see that in this case $h^{(\mathrm{pps})}(F)=2$ whereas $h^{\text {(botk) }}(F) \geq(n / 2+k) / k$

Computation: When the data has the form of elements $\left(x, w_{x}\right)$ with unique keys and $f_{x}=f\left(w_{x}\right)$ for a set of functions $F$, then short of further structural assumptions on $F$, the sampling algorithm that computes $S^{(F)}$ must apply all functions $f \in F$ to all elements. The computation is thus $\Omega(|F| n)$ and can be $O\left(|F| n+\left|S^{(F)}\right| \log k\right)$ time by identifying for each $f \in F$, the $k$ keys with smallest $f$-seed $(x)$. In the sequel we will see examples of large or infinite sets $F$ but with special structure that allows us to efficiently compute a multi-objective sample.

\section{The Sampling Closure}

We define the sampling closure $\bar{F}$ of a set of functions $F$ to be the set of all functions $f$ such that for all $k$ and for all $H$, the estimate of $\operatorname{sum}(f ; H)$ from $S^{(F, k)}$ has the CV bound of Theorem 2.1. Note that this definitions is with respect to uniform guarantees (same size parameter $k$ for all objectives). We show that the closure of $F$ contains all non-negative linear combinations of functions from $F$.

Theorem 5.1. Any $f=\sum_{g \in F} \alpha_{g} g$ where $\alpha_{g} \geq 0$ is in $\bar{F}$.

Proof. We first consider pps samples, where we establish the stronger claim $S^{(F \cup\{f\}, k)}=S^{(F, k)}$, or equivalently,

$$
\text { for all keys } x, p_{x}^{(f, k)} \leq p_{x}^{(F, k)} \text {. }
$$

For a function $g$, we use the notation $g(\mathcal{X})=\sum_{y} g_{y}$, and recall that $p_{x}^{(g, k)}=\min \left\{1, k \frac{g_{x}}{g(\mathcal{X})}\right\}$. We first consider $f=c g$ for some $g \in F$. In this case, $p_{x}^{(f, k)}=p_{x}^{(g, k)} \leq p_{x}^{(F, k)}$ and 8 follows. To complete the proof, it suffices to establish (8) for $f=g^{(1)}+g^{(2)}$ such that $g^{(1)}, g^{(2)} \in F$. Let $c$ be such that $\frac{g_{x}^{(2)}}{g^{(2)}(\mathcal{X})}=c \frac{g_{x}^{(1)}}{g^{(1)}(\mathcal{X})}$ we can assume WLOG that $c \leq 1$ (otherwise reverse $g^{(1)}$ and $\left.g^{(2)}\right)$. For convenience denote $\alpha=g^{(2)}(\mathcal{X}) / g^{(1)}(\mathcal{X})$. Then we can write

$$
\begin{aligned}
\frac{f_{x}}{f(\mathcal{X})} & =\frac{g_{x}^{(1)}+g_{x}^{(2)}}{g^{(1)}(\mathcal{X})+g^{(2)}(\mathcal{X})} \\
& =\frac{(1+c \alpha) g_{x}^{(1)}}{(1+\alpha) g^{(1)}(\mathcal{X})}=\frac{1+c \alpha}{1+\alpha} \frac{g_{x}^{(1)}}{g^{(1)}(\mathcal{X})} \\
& \leq \frac{g_{x}^{(1)}}{g^{(1)}(\mathcal{X})}=\max \left\{\frac{g_{x}^{(1)}}{g^{(1)}(\mathcal{X})}, \frac{g_{x}^{(2)}}{g^{(2)}(\mathcal{X})}\right\}
\end{aligned}
$$

Therefore $p_{x}^{(f, k)} \leq \max \left\{p_{x}^{\left(g^{(1)}, k\right)}, p_{x}^{\left(g^{(2)}, k\right)}\right\} \leq p_{x}^{(F, k)}$.

The proof for multi-objective bottom- $k$ samples is more involved, and deferred to the full version. Note that the multiobjective bottom- $k$ sample $S^{(F, k)}$ may not include a bottom- $k$ sample $S^{(f, k)}$, but it is still possible to bound the CV.

\section{The UNIVERSAL SAMple FOR MONOtONE STATISTICS}

In this section we consider the (infinite) set $M$ of all monotone non-decreasing functions and the objectives $(f, k)$ for all $f \in M$.

We show that the multi-objective pps and bottom- $k$ samples $S^{(M, k)}$, which we refer to as the universal monotone sample, are larger than a single dedicated weighted sample by at most a logarithmic factor in the number of keys. We will also show that this is tight. We will also present efficient universal monotone bottom- $k$ sampling scheme for streamed or distributed data.

We take the following steps. We consider the multi-objective sample $S^{\text {(thresh, } k \text { ) }}$ for the set thresh of all threshold functions ${\text { (recall that } \operatorname{thresh}_{T}(x)=1 \text { if } x \geq T \text { and thresh }}_{T}=0$ otherwise). We express the inclusion probabilities $p_{x}^{\text {(thresh, } k \text { ) }}$ and bound the sample size. Since all threshold functions are monotone, thresh $\subset M$. We will establish that $S^{(\text {thresh, } k)}=S^{(M, k)} 4 \mathrm{We}$ start with the simpler case of pps and then move on to bottom- $k$ samples.

\subsection{Universal monotone pps}

Theorem 6.1. Consider a data set $\mathcal{D}=\left\{\left(x, w_{x}\right)\right\}$ of $n$ keys and the sorted order of keys $x$ by non-increasing $w_{x}$. Then a key $x$ that is in position $i$ in the sorted order has base multi-objective pps probability

$$
p_{x}^{(\text {thresh }, 1)}=p_{x}^{(M, 1)} \leq 1 / i .
$$

When all keys have unique weights, equality holds.

Proof. Consider the function thresh ${ }_{w_{x}}$. The function has weight 1 on all the $\geq i$ keys with weight $\geq w_{x}$. Therefore, the base pps probability is $p_{x}^{\left.\text {(thresh } w_{x}, 1\right)} \leq 1 / i$. When the keys have unique weights then there are exactly $i$ keys $y$ with weight $w_{y} \geq w_{x}$ and we have $p_{x}^{\left.\text {(thresh }_{w_{x}}, 1\right)}=1 / i$. If we consider all threshold

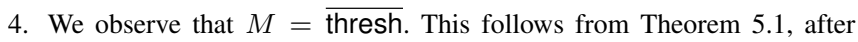
noticing that any $f \in M$ can be expressed as a non-negative combination of threshold functions

$$
f(y)=\int_{0}^{\infty} \alpha(T) \underset{T}{\operatorname{thresh}}(y) d T,
$$

for some function $\alpha(T) \geq 0$. We establish here the stronger relation $S^{(\text {thresh, } k)}=S^{(M, k)}$. 
functions, then $p_{x}^{\left(\text {thresh }_{T}, 1\right)}=0$ when $T>w_{x}$ and $p_{x}^{\left(\text {thresh }_{T}, 1\right)} \leq$ $1 / i$ when $T \leq w_{x}$. Therefore,

$$
p_{x}^{(\text {thresh }, 1)}=\max _{T} p_{x}^{\left(\text {thresh }_{T}, 1\right)}=p_{x}^{\left(w_{x}, 1\right)}=1 / i .
$$

We now consider an arbitrary monotone function $f_{x}=f\left(w_{x}\right)$. From monotonicity there are at least $i$ keys with $f_{y} \geq f_{x}$ therefore, $f_{x} / \sum_{y} f_{y} \leq 1 / i$. Thus, $p_{x}^{(f, 1)}=f_{x} / \sum_{y} f_{y} \leq 1 / i$ and

$$
p_{x}^{(M, 1)}=\max _{f \in M} p_{x}^{(f, 1)} \leq 1 / i
$$

There is a simple main-memory sampling scheme where we sort the keys, compute the probabilities $p^{(M, 1)}$ and then $p^{(M, k)}=\min \left\{1, k p^{(M, 1)}\right\}$ and compute a sample accordingly. We next present universal monotone bottom- $k$ samples and sampling schemes that are efficient on streamed or distributed data.

\subsection{Universal monotone bottom- $k$}

Theorem 6.2. Consider a data set $\mathcal{D}=\left\{\left(x, w_{x}\right)\right\}$ of $n$ keys. The universal monotone bottom- $k$ sample has expected size $E\left[\left|S^{(M, k)}\right|\right] \leq k \ln n$ and can be computed using $O(n+$ $k \log n \log k)$ operations.

For a particular $T$, the bottom- $k$ sample $S^{\left(\text {thresh }_{T}, k\right)}$ is the set of $k$ keys with smallest $u_{x}$ among keys with $w_{x} \geq T$. The set of keys in the multi-objective sample is $S^{(\text {thresh, } \bar{k})}=$ $\bigcup_{T>0} S^{\left(\text {thresh }_{T}, k\right)}$. We show that a key $x$ is in the multi-objective sample for thresh if and only if it is in the bottom- $k$ sample for thresh $_{w_{x}}$ :

Lemma 6.1. Fixing $\left\{u_{y}\right\}$, for any key $x$,

$$
x \in S^{(\text {thresh,k) }} \Longleftrightarrow x \in S^{\left(\text {thresh }_{w_{x}}, k\right)} .
$$

Proof. Consider the position $t(x, T)$ of $x$ in an ordering of keys $y$ induced by thresh $T_{T}$-seed $(y)$. We claim that if for a key $y$ we have thresh $T^{\text {-seed }(x)}<\operatorname{thresh}_{T}$-seed $(y)$ for some $T>0$, this must hold for $T=w_{x}$. The claim can be established by separately considering $w_{y} \geq w_{x}$ and $w_{y}<w_{x}$. The claim implies that $t(x, T)$ is minimized for $T=w_{x}$.

We now consider the auxiliary keys $Z$ associated with this sample. Recall that these keys are not technically part of the sample but the information $\left(u_{x}, w_{x}\right)$ for $x \in Z$ is needed in order to compute the conditional inclusion probabilities $p_{x}^{\text {(thresh, } k \text { ) }}$ for $x \in S$. Note that it follows from Lemma 6.1 that for all keys $x$, $p_{x}^{(\text {thresh }, k)}=p_{x}^{\left.\text {(thresh }_{w_{x}}, k\right)}$. For a key $x$, let $Y_{x}=\left\{y \neq x \mid w_{y} \geq\right.$ $\left.w_{x}\right\}$ be the set of keys other than $x$ that have weight that is at least that of $x$. Let $y_{x}$ be the key with $k$ th smallest $u_{x}$ in $Y_{x}$, when $\left|Y_{x}\right| \geq k$. The auxiliary keys are $Z=\left\{y_{x} \mid x \in S\right\} \backslash S$. A key $x$ is included in the sample with probability 1 if $y_{x}$ is not defined (which means it has one of the $k$ largest weights). Otherwise, it is (conditionally) included if and only if $u_{x}<u_{y_{x}}$. To compute the inclusion probability $p_{x}^{(\text {thresh }, k)}$ from $S \cup Z$, we do as follows. If there are $k$ or fewer keys in $S \cup Z$ with weight that is at most $w_{x}$, then $p_{x}^{\text {(thresh, } k \text { ) }}=1$ (For correctness, note that in this case all keys with weight $\geq w_{x}$ would be in $S$.) Otherwise, observe that $y_{x}$ is the key with $(k+1)$ th smallest $u$ value in $S \cup Z$ among all keys $y$ with $w_{y} \geq w_{x}$. We compute $y_{x}$ from the sample and use $p_{x}^{(\text {thresh }, k)}=u_{y_{x}}$. Note that when weights are unique, $Z=\emptyset$.
The definition of $S^{(\text {thresh, } k)}$ is equivalent to that of an AllDistances Sketch (ADS) computed with respect to weights $w_{x}$ (as inverse distances) [7], [8], and we can apply some algorithms and analysis. In particular, we obtain that $\mathrm{E}\left[\left|S^{(\text {thresh }, k)}\right|\right] \leq k \ln n$ and the size is well-concentrated around this expectation. The argument is simple: Consider keys ordered by decreasing weight. The probability that the $i$ th key has one of the $k$ smallest $u_{x}$ values, and thus is a member of $S^{\text {(thresh, } k \text { ) }}$ is at most $\min \{1, k / i\}$. Summing the expectations over all keys we obtain $\sum_{i=1}^{n} \min \{1, k / i\}<k \ln n$. We shall see that the bound is asymptotically tight when weights are unique. With repeated weights, however, the sample size can be much smaller ${ }^{5}$

Lemma 6.2. For any data set $\left\{\left(x, w_{x}\right)\right\}$, when using the same randomization $\left\{u_{x}\right\}$ to generate both samples, $S^{(M, k)}=$ $S^{(\text {thresh,k) }}$.

Proof. Consider $f \in M$ and the samples obtained for some fixed randomization $u_{y}$ for all keys $y$. Suppose that a key $x$ is in the bottom- $k$ sample $S^{(f, k)}$. By definition, we have that $f$ seed $(x)=r_{x} / f\left(w_{x}\right)$ is among the $k$ smallest $f$-seeds of all keys. Therefore, it must be among the $k$ smallest $f$-seeds in the set $Y$ of keys with $w_{y} \geq w_{x}$. From monotonicity of $f$, this implies that $r_{x}$ must be one of the $k$ smallest in $\left\{r_{y} \mid y \in Y\right\}$, which is the same as $u_{x}$ being one of the $k$ smallest in $\left\{u_{y} \mid y \in Y\right\}$. This implies that $x \in S^{\left(\text {thresh }_{w_{x}}, k\right)}$.

\subsection{Estimation quality}

The estimator (5) with the conditional inclusion probabilities $p_{x}^{(M, k)}$ generalizes the HIP estimator of [8] to sketches computed for non-unique weights. Theorem 4.1 implies that for any $f \in M$ and $H, \mathrm{C} V[\widehat{\operatorname{sum}}(f ; H)] \leq \frac{1}{\sqrt{q^{(f)}(H)(k-1)}}$. When weights are unique and we estimate statistics over all keys, we have the tighter bound $\mathrm{C} V[\widehat{\operatorname{sum}}(f ; \mathcal{X})] \leq \frac{1}{\sqrt{2 k-1}}[8]$.

\subsection{Sampling algorithms}

The samples, including the auxiliary information, are composable. Composability holds even when we allow multiple elements to have the same key $x$ and interpret $w_{x}$ to be the maximum weight over elements with key $x$. To do so, we use a random hash function to generate $u_{x}$ consistently for multiple elements with the same key. To compose multiple samples, we take a union of the elements, replace multiple elements with same key with the one of maximum weight, and apply a sampling algorithm to the set of remaining elements. The updated inclusion probabilities can be computed from the composed sample.

We present two algorithms that compute the sample $S^{(M, k)}$ along with the auxiliary keys $Z$ and the inclusion probabilities $p_{x}^{(M, k)}$ for $x \in S^{(M, k)}$. The algorithms process the elements either in order of decreasing $w_{x}$ or in order of increasing $u_{x}$. These two orderings may be suitable for different applications and it is worthwhile to present both: In the related context of alldistances sketches, both ordering on distances were used [7], [26], [3], [8]. The algorithms are correct when applied to any set of $n$ elements that includes $S \cup Z$. Recall that the inclusion probability $p_{x}^{(M, k)}$ is the $k$ th smallest $u_{x}$ among keys with $w_{x} \leq w$. Therefore, all keys with the same weight have the same inclusion

5. The sample we would obtain with repeated weights is always a subset of the sample we would have obtained with tie breaking. In particular, the sample size can be at most $k$ times the number of unique weights. 
probability. For convenience, we thus express the probabilities as a function $p(w)$ of the weights.

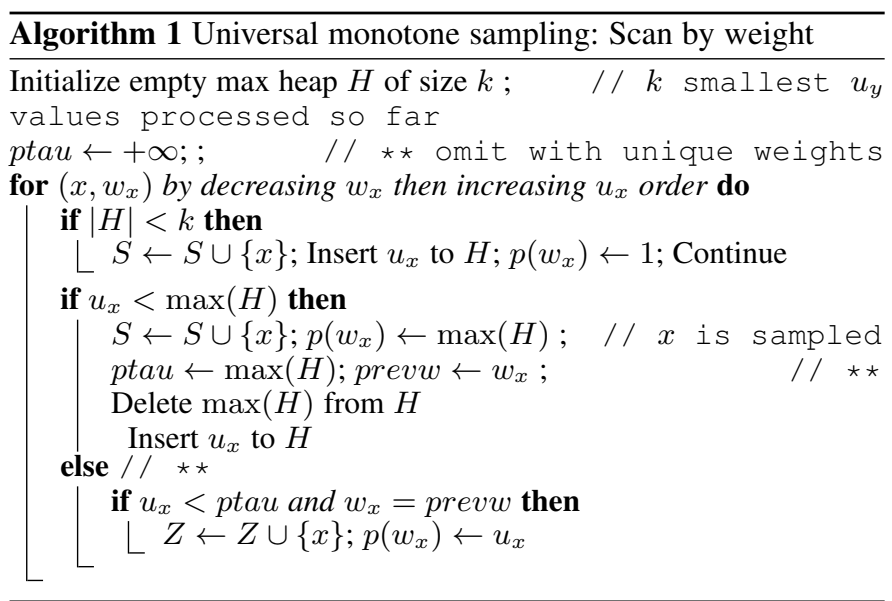

Algorithm 1 processes keys by order of decreasing weight, breaking ties by increasing $u_{x}$. We maintain a max-heap $H$ of the $k$ smallest $u_{y}$ values processed so far. When processing a current key $x$, we include $x \in S$ if $u_{x}<\max (H)$. If including $x$, we delete $\max (H)$ and insert $u_{x}$ into $H$. Correctness follows from $H$ being the $k$ smallest $u$ values of keys with weight at least $w_{x}$. When weights are unique, the probability $p\left(w_{x}\right)$ is the $k$ th largest $u$ value in $H$ just before $x$ is inserted. When weights are not unique, we also need to compute $Z$. To do so, we track the previous $\max (H)$, which we call ptau. If the current key $x$ has $u_{x} \in(\max (H), p t a u)$, we include $x \in Z$. It is easy to verify that in this case, $p\left(w_{x}\right)=u_{x}$. Note that the algorithm may overwrite $p(w)$ multiple times, as keys with weight $w$ are inserted to the sample or to $Z$.

Algorithm 2 processes keys in order of increasing $u_{x}$. The algorithm maintains a min heap $H$ of the $k$ largest weights processed so far. With unique weights, the current key $x$ is included in the sample if and only if $w_{x}>\min (H)$. If $x$ is included, we delete from the heap $H$ the key with weight $\min (H)$ and insert $x$. When weights are not unique, we also track the weight $w$ of the previous removed key from $H$. When processing a key $x$ then if $w_{x}=\min (H)$ and $w_{x}>$ prevw then $x$ is inserted to $Z$.

The computed inclusion probabilities $p(w)$ with unique weights is $u_{y}$, were $y$ is the key whose processing triggered the deletion of the key $x$ with weight $w$ from $H$. To establish correctness, consider the set $H$, just after $x$ is deleted. By construction, $H$ contains the $k$ keys with weight $w_{y}<w_{x}$ that have smallest $u$ values. Therefore, $p\left(w_{x}\right)=\max _{y \in H} u_{y}$. Since we process keys by increasing $u_{y}$, this maximum $u$ value in $H$ is the value of the most recent inserted key, that is, the key $y$ which triggered the removal of $x$. Finally, the keys that remain in $H$ in the end are those with the $k$ largest weights. The algorithms correctly assigns $p\left(w_{x}\right)=1$ for these keys.

When multiple keys can have the same weight, then $p(w)$ is the minimum of $\max _{y \in H} u_{y}$ after the first key of weight $w$ is evicted, and the minimum $u_{z}$ of a key $z$ with $w_{z}=w$ that was not sampled. If the minimum is realized at such a key $z$, that key is included in $Z$, and the algorithm set $p(w)$ accordingly when $z$ is processed. If $p(w)$ is not set already when the first key of weight $w$ is deleted from $H$, the algorithm correctly assigns $p(w)$ to be $\max _{y \in H} u_{y}$. After all keys are processed, $p\left(w_{x}\right)$ is set for remaining keys $x \in H$ where a key with the same weight was previously deleted from $H$. Other keys are assigned $p(w)=1$.

We now analyze the number of operations. With both algorithms, the cost of processing a key is $O(1)$ if the key is not inserted and $O(\log k)$ if the key is included in the sample. Using the bound on sample size, we obtain a bound of $O(n+k \ln n \log k)$ on processing cost. The sorting requires $O(n \log n)$ computation, which dominates the computation (since typically $k \ll n$ ). When $u_{x}$ are assigned randomly, however, we can generate them with a sorted order by $u_{x}$ in $O(n)$ time, enabling a faster $O(n+k \log k \log n)$ computation.

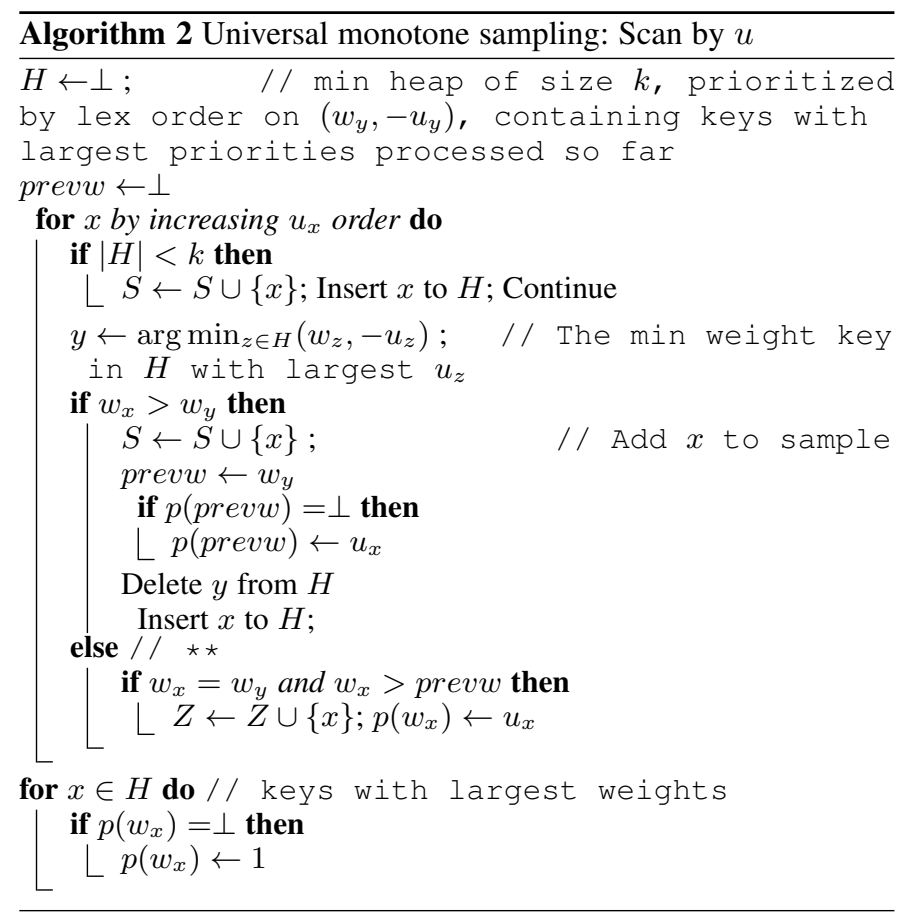

\subsection{Lower bound on sample size}

We now show that the worst-case factor of $\ln n$ on the size of universal monotone sample is in a sense necessary. It suffices to show this for threshold functions:

Theorem 6.3. Consider data sets where all keys have unique weights. Any sampling scheme with a nonnegative unbiased estimator that for all $T>0$ and $H$ has

$$
C V\left[\widehat{\operatorname{sum}}\left(\text { thresh }_{T} H\right)\right] \leq 1 / \sqrt{q^{\left(\text {thresh }_{T}\right)}(H) k},
$$

must have samples of expected size $\Omega(k \ln n)$.

Proof. We will use Theorem 3.1 which relates estimation quality to sampling probabilities. Consider the key $x$ with the $i$ th heaviest weight. Applying Theorem 3.1 to $x$ and thresh $w_{x}$ we obtain that $p_{x} \geq \frac{k}{k+i}$

Summing the sampling probabilities over all keys $i \in$ $[n]$ to bound the expected sample size we obtain $\sum_{x} p_{x} \geq$ $k \sum_{i=1}^{n} \frac{1}{k+i}=k\left(H_{n}-H_{k}\right) \approx k(\ln n-\ln k)$.

\section{The UNIVERSAL CAPPING SAMPLE}

An important strict subset of monotone functions is the set $C=\left\{\operatorname{cap}_{T} \mid T>0\right\}$ of capping functions. We study the multi-objective bottom- $k$ sample $S^{(C, k)}$, which we refer to as the 
universal capping sample. From Theorem 5.1 the closure of $C$ includes all functions of the form $f(y)=\int_{0}^{\infty} \alpha(T) \operatorname{cap}_{T}(y) d T$, for some $\alpha(T) \geq 0$. This is the set of all non-decreasing concave functions with at most a linear growth, that is $f(w)$ that satisfy $\frac{d f}{d w} \leq 1$ and $\frac{d^{2} f}{d w} \leq 0$.

We show that the sample $S^{(C, k)}$ can be computed using $O(n+$ $k \log n \log k$ ) operaions from any $D^{\prime} \subset D$ that is superset of the keys in $S^{(C, k)}$. We start with properties of $S^{(C, k)}$ which we will use to design our sampling algorithm. For a key $x$, let $h_{x}$ be the number $h$ of keys with $w_{y} \geq w_{x}$ and $u_{y}<u_{x}$. Let $\ell_{x}$ be the number of keys $y$ with $w_{y}<w_{x}$ and $r_{y} / w_{y}<r_{x} / w_{x}$.

For a key $x$ and $T>0$ and fixing the assignment $\left\{u_{y}\right\}$ for all keys $y$, let $t(x, T)$ be the position of $\operatorname{cap}_{T}$-seed $(x)$ in the list of

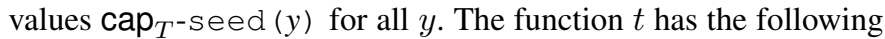
properties:

Lemma 7.1. For a key $x, t(x, T)$ is minimized for $T=w_{x}$. Moreover, $t(x, T)$ is non-decreasing for $T \geq w_{x}$ and nonincreasing for $T \leq w_{x}$.

Proof. We can verify that for any key $y$ such that there is a $T>0$ such that the $\operatorname{cap}_{T}$-seed $(x)<\operatorname{cap}_{T}$-seed $(y)$, we must have $\operatorname{cap}_{w_{x}}$-seed $(x)<\operatorname{cap}_{w_{x}}$-seed $(y)$. Moreover, the set of $T$ values where $\operatorname{cap}_{T}$-seed $(x)<\operatorname{cap}_{T}$-seed $(y)$ is an interval which contains $T=w_{x}$. We can establish the claim by separately considering the cases $w_{y} \geq w_{x}$ and $w_{y}<w_{x}$.

As a corollary, we obtain that a key is in the universal capping sample only if it is in the bottom- $k$ sample for $\operatorname{cap}_{w_{x}}$ :

Corollary 7.2. Fixing $\left\{u_{y}\right\}$, for any key $x$,

$$
x \in S^{(C, k)} \Longleftrightarrow x \in S^{\left(\operatorname{cap}_{w_{x}}, k\right)} .
$$

Lemma 7.3. Fixing the assignment $\left\{u_{x}\right\}$,

$$
x \in S^{(C, k)} \Longleftrightarrow \ell_{x}+h_{x}<k .
$$

Proof. From Lemma 7.1, a key $x$ is in a bottom- $k$ cap $_{T}$ sample for some $T$ if and only if it is in the bottom- $k$ sample for cap $w_{x}$. The keys with a lower $\operatorname{cap}_{w_{x}}$-seed than $x$ are those with $w_{y} \geq w_{x}$ and $u_{y}<u_{x}$, which are counted in $h_{x}$, and those with $w_{y}<w_{x}$ and $r_{y} / w_{y}<r_{x} / w_{x}$, which are counted in $\ell_{x}$. Therefore, a key $x$ is in $S^{\left(\operatorname{cap}_{w_{x}}, k\right)}$ if and only if there are fewer than $k$ keys with a lower cap $_{w_{x}}$-seed, which is the same as having $h_{x}+\ell_{x}<k$.

For reference, a key $x$ is in the universal monotone sample $S^{(M, k)}$ if and only if it satisfies the weaker condition $h_{x}<k$.

Lemma 7.4. A key $x$ can be auxiliary only if $\ell_{x}+h_{x}=k$

Proof. A key $x$ is auxiliary (in the set $Z$ ) only if for some $y \in S$, it has the $k$ th smallest $\operatorname{cap}_{w_{y}}$-seed among all keys other than $y$. This means it has the $(k+1)$ th smallest cap $w_{y}$-seed.

The number of keys with seed smaller than seed $(x)$ is minimized for $T=w_{x}$. If the $\operatorname{cap}_{w_{x}}$-seed of $x$ is one of the $k$ smallest ones, it is included in the sample. Therefore, to be auxiliary, it must have the $(k+1)$ th smallest seed.

\subsection{Sampling algorithm}

We are ready to present our sampling algorithm. We first process the data so that multiple elements with the same key are replaced with with the one with maximum weight. The next step is to identify all keys $x$ with $h_{x} \leq k$. It suffices to compute $S^{(M, k)}$ of the data with the auxiliary keys. We can apply a variant of
Algorithm 11. We process the keys in order of decreasing weight, breaking ties by increasing rank, while maintaining a binary search tree $H$ of size $k$ which contains the $k$ lowest $u$ values of processed keys. When processing $x$, if $u_{x}>\max (H)$ then $h_{x}>k$, and the key $x$ is removed. Otherwise, $h_{x}$ is the position of $u_{x}$ in $H$, and $u_{x}$ is inserted to $H$ and $\max (H)$ removed from $H$. We now only consider keys with $h_{x} \leq k$. Note that in expectation there are at most $k \ln n$ such keys.

The algorithm then computes $\ell_{x}$ for all keys with $\ell_{x} \leq k$. This is done by scanning keys in order of increasing weight, tracking in a binary search tree structure $H$ the (at most) $k$ smallest $r_{y} / w_{y}$ values. When processing $x$, if $r_{x} / w_{x}<\max (H)$, then $\ell_{x}$ is the position of $r_{x} / w_{x}$ in $H$. We then delete $\max (H)$ and insert $r_{x} / w_{x}$.

Keys that have $\ell_{x}+h_{x}<k$ then constitute the sample $S$ and keys with $\ell_{x}+h_{x}=k$ are retained as potentially being auxiliary.

Finally, we perform another pass on the sampled and potentially auxiliary keys. For each key $x$, we determine the $k+1$ th smallest $\operatorname{cap}_{w_{x}}$-seed, which is $\tau^{\left(\operatorname{cap}_{w_{x}}, k\right)}$. Using Corollary 7.2 we can use (3) to compute $p_{x}^{\left(\operatorname{cap}_{w_{x}}, k\right)}=p_{x}^{(C, k)}$. At the same time we can also determine the precise set of auxiliary keys by removing those that are not the $(k+1)$ th smallest seed for any $\operatorname{cap}_{w_{x}}$ for $x \in S$.

\subsection{Size of $S^{(C, k)}$}

The sample $S^{(C, k)}$ is contained in $S^{(M, k)}$, but can be much smaller. Intuitively, this is because two keys with similar, but not necessarily identical, weights are likely to have the same relation between their $f$-seeds across all $f \in C$. This is not true for $M$ : For a threshold $T$ between the two weights, the thresh ${ }_{T}$-seed would always be lower for the higher weight key whereas the relation for lower $T$ value can be either one with almost equal probabilities. In particular, we obtain a bound on $\left|S^{(C, k)}\right|$ which does not depend on $n$ :

\section{Theorem 7.1.}

$$
E\left[\left|S^{(C, k)}\right|\right] \leq e k \ln \frac{\max _{x} w_{x}}{\min _{x} w_{x}} .
$$

Proof. Consider a set of keys $Y$ such that $\frac{\max _{x \in Y} w_{x}}{\min _{x \in Y} w_{x}}=\rho$. We show that the expected number of keys $x \in Y$ that for at least one $T>0$ have one of the bottom- $k \operatorname{cap}_{T}$-seeds is at most $\rho k$. The claim then follows by partitioning keys to $\ln \frac{\max _{x} w_{x}}{\min _{x} w_{x}}$ groups where weights within each group vary by at most a factor of $e$ and then noticing that the bottom- $k$ across all groups must be a subset of the union of bottom- $k$ sets within each group.

We now prove the claim for $Y$. Denote by $\tau$ the $(k+1)$ th smallest $r_{x}$ value of $x \in Y$. The set of $k$ keys with $r_{x}<\tau$ are the bottom- $k$ sample for cap $T \leq \min _{x \in Y} w_{x}$. Consider a key $y$. From Lemma 7.1. we have $y \in S^{(C, k)}$ only if $y \in S^{\left(\mathrm{cap}_{w_{y}}, k\right)}$. A necessary condition for the latter is that $r_{y} / w_{y}<\tau / \min _{x \in Y} w_{x}$. This probability is at most

$$
\begin{aligned}
& \underset{u_{y} \sim U[0,1]}{\operatorname{Pr}}\left[\frac{r_{y}}{w_{y}}<\frac{\tau}{\min _{x \in Y} w_{x}}\right] \\
& \leq \frac{w_{y}}{\min _{x \in Y} w_{x}} \underset{u_{x} \sim U[0,1]}{\operatorname{Pr}}\left[r_{y}<\tau\right] \leq \rho \frac{k}{|Y|}
\end{aligned}
$$

Thus, the expected number of keys that satisfy this condition is at most $\rho k$. 


\section{Metric objectives}

In this section we discuss the application of multi-objective sampling to additive cost objectives. The formulation has a set of keys $X$, a set of models $\mathcal{Q}$, and a nonnegative cost function $c(Q, x)$ of servicing $x \in X$ by $Q \in \mathcal{Q}$. In metric settings, the keys $X$ are points in a metric space $M, Q \in \mathcal{Q}$ is a configuration of facilities (that can also be points $Q \subset M)$, and $c(Q, x)$ is distance-based and is the cost of servicing $x$ by $Q$. For each $Q \in \mathcal{Q}$ we are interested in the total cost of servicing $X$ which is

$$
c(Q, X)=\sum_{x \in X} c(Q, x) .
$$

A concrete example is the $k$-means clustering cost function, where $Q$ is a set of points (centers) of size $k$ and $c(Q, x)=$ $\min _{q \in Q} d(q, x)^{2}$.

In this formulation, we are interested in computing a small summary of $X$ that would allow us to estimate $c(Q, X)$ for each $Q \in \mathcal{Q}$. Such summaries in a metric setting are sometimes referred to as coresets [1]. Multi-objective samples can be used as such a summary. Each $Q \in \mathcal{Q}$ has a corresponding function $f_{x} \equiv c(Q, x)$. A multi-objective sample for the set $F$ of all the functions for $Q \in \mathcal{Q}$ allows us to estimate $\operatorname{sum}(f)=c(Q, X)$ for each $Q$. In particular, a sample of size $h(F) \epsilon^{-2}$ allows us to estimate $c(Q, X)$ for each $Q \in \mathcal{Q}$ with $\mathrm{CV}$ at most $\epsilon$ and good concentration.

The challenges, for a domain of such problems, are to

- Upper-bound the multi-objective overhead $h(F)$ as a function of parameters of the domain $(|X|, c$, structure of $Q \in \mathcal{Q}$ ). The overhead is a fundamental property of the problem domain.

- Efficiently compute upper bounds $\mu_{x}$ on the multiobjective sampling probabilities $p^{(F, 1)}$ so that the sum $\sum_{x} \mu_{x}$ is not much larger than $h(F)$. We are interested in obtaining these bounds without enumerating over $Q \in \mathcal{Q}$ (which can be infinite or very large).

Recently, we [6] applied multi-objective sampling to the problem of centrality estimation in metric spaces. Here $M$ is a general metric space, $X \subset M$ is a set of points, each $Q$ is a single point in $M$, and the cost functions is $c(Q, x)=d(Q, x)^{p}$. The centrality $Q$ is the sum $\sum_{x} c(Q, x)$. We established that the multi-objective overhead is constant and that upper bound probabilities (with constant overhead) can be computed very efficiently using $O(|X|)$ distance computations. More recently [10], we generalized the result to the $k$-means objective where $Q$ are subsest of size at most $k$ and establish that the overhead is $O(k)$.

\section{ForEach, ForAll}

Our multi-objective sampling probabilities provide statistical guarantees that hold for each $f$ and $H$ : Theorem 4.1 states that the estimate $\widehat{\operatorname{sum}}(f ; H)$ has the $\mathrm{CV}$ and concentration bounds over the sample distribution $S \sim \boldsymbol{p}$ (sample $S$ that includes each $x \in \mathcal{X}$ independently (or VarOpt) with probability $p_{x}$ ).

In this section we focus on uniform per-objective guarantees $\left(k_{f}=k\right.$ for all $\left.f \in F\right)$ and statistics $\operatorname{sum}(f ; \mathcal{X})=\operatorname{sum}(f)$ over the full data set. For $F$ and probabilities $\boldsymbol{p}$, we define the ForEach Normalized Mean Squared Error (NMSE):

$$
\operatorname{NMSE}_{e}(F, \boldsymbol{p})=\max _{f \in F} \mathrm{E}_{S \sim \boldsymbol{p}}\left(\frac{\widehat{\operatorname{sum}}(f)}{\operatorname{sum}(f)}-1\right)^{2},
$$

and the ForAll NMSE:

$$
\operatorname{NMSE}_{a}(F, \boldsymbol{p})=\mathrm{E}_{S \sim \boldsymbol{p}} \max _{f \in F}\left(\frac{\widehat{\operatorname{sum}}(f)}{\operatorname{sum}(f)}-1\right)^{2} .
$$

The respective normalized root MSE (NRMSE) are the squared roots of the NMSE. Note that ForAll is stronger than ForEach as it requires a simultaneous good approximation of $\operatorname{sum}(f)$ for all $f \in F$ :

$$
\forall \boldsymbol{p}, \operatorname{NMSE}_{e}(F, \boldsymbol{p}) \leq \operatorname{NMSE}_{a}(F, \boldsymbol{p}) .
$$

We are interested in the tradeoff between the expected size of a sample, which is $\operatorname{sum}(\boldsymbol{p}) \equiv \sum_{x} p_{x}$, and the NRMSE. The multi-objective pps probabilities are such that for all $\ell>1$, $\operatorname{NMSE}_{e}\left(F, \boldsymbol{p}^{(F, \ell)}\right) \leq 1 / \ell$.

For a parameter $\ell \geq 1$, we can also consider the ForAll error $\operatorname{NMSE}_{a}\left(F, \boldsymbol{p}^{(F, \ell)}\right)$ and ask for a bound on $\ell$ so that the $\mathrm{NRMSE}_{a} \leq \epsilon$. A union-bound argument established that $\ell=\epsilon^{-2} \log |\bar{F}|$ always suffices. Moreover, when $F$ is the sampling closure of a smaller subset $F^{\prime}$, then $\ell=\epsilon^{-2} \log \left|F^{\prime}\right|$ suffices. If we only bound the maximum error on any subset of $F$ of size $m$, we can use $\ell=\epsilon^{-2} \log m$. When $F$ is the set of all monotone functions over $n$ keys, then $\ell=O\left(\epsilon^{-2} \log \log n\right)$ suffices. To see this intuitively, recall that it suffices to consider all threshold functions since all monotone functions are nonnegative combinations of threshold functions. There are $n$ threshold functions but these functions have $O(\log n)$ points where the value significantly changes by a factor.

We provide an example of a family $F$ where the sample-size gap between $\mathrm{NMSE}_{e}$ and $\mathrm{NMSE}_{a}$ is linear in the support size. Consider a set of $n$ keys and define $f$ for each subset of $n / 2$ keys so that $f$ is uniform on the subset and 0 outside it. The multi-objective base pps sampling probabilities are $p_{x}^{(F, 1)}=2 / n$ for all $x$ and hence the overhead is $h(F)=2$. Therefore, $\boldsymbol{p}$ of size $2 \epsilon^{-2}$ has $\operatorname{NRMSE}_{e}(F, \boldsymbol{p}) \leq \epsilon$. In contrast, any $\boldsymbol{p}$ with $\operatorname{NRMSE}_{a}(F, \boldsymbol{p}) \leq 1 / 2$ must contain at least one key from the support of each $f$ in (almost) all samples, implying expected sample size that is at least $n / 2$.

When we seek to bound $\mathrm{NRMSE}_{e}$, probabilities of the form $\boldsymbol{p}^{(F, \ell)}$ essentially optimize the size-quality tradeoff. For $\mathrm{NRMSE}_{a}$, however, the minimum size $\boldsymbol{p}$ that meets a certain error $\operatorname{NRMSE}_{a}(F, \boldsymbol{p}) \leq \epsilon$ can be much smaller than the minimum size $\boldsymbol{p}$ that is restricted to the form $\boldsymbol{p}^{(F, \ell)}$. Consider $\epsilon>0$ and a set $F$ that has $k$ parts $F_{i}$ with disjoint supports of equal sizes $n / k$. All the parts $F_{i}$ except $F_{1}$ have a single $f$ that is uniform on the support, which means that with uniform $p$ of size $\epsilon^{-2}$ we have, $\operatorname{NRMSE}_{e}\left(F_{i}, \boldsymbol{p}\right)=\operatorname{NRMSE}_{a}\left(F_{i}, \boldsymbol{p}\right)=\epsilon$. The part $F_{1}$ has similar structure to our previous example which means that any $\boldsymbol{p}$ that has $\operatorname{NRMSE}_{a}\left(F_{1}, \boldsymbol{p}\right) \leq 1 / 2$ has size at least $n /(2 k)$ wheras a uniform $\boldsymbol{p}$ of size $2 \epsilon^{-2}$ has $\operatorname{NRMSE}_{e}\left(F_{1}, \boldsymbol{p}\right)=\epsilon$. The multiobjective base sampling probabilities are therefore $p^{(F, 1)}=k / n$ for keys in the supports of $F_{i}$ where $i>1$ and $p^{(F, 1)}=2 k / n$ for keys in the support of $F_{1}$ and thus the overhead is $k+1$. The minimum size $\boldsymbol{p}$ for $\operatorname{NRMSE}_{a}(F, \boldsymbol{p})=1 / 2$ must have value at least $1 / 2$ for keys in the support of $F_{1}$ and value about $(k / n) \log (k)$ for other keys (having ForAll requirement for each part and a logarithmic factor due to a (tight) union bound). Therefore the sample size is $O(n / k+k \log k)$. In contrast, To have $\operatorname{NRMSE}_{a}\left(F, \boldsymbol{p}^{(F, \ell)}\right)=1 / 2$, we have to use $\ell=\Omega(n / k)$, obtaining a sample size of $\Omega(n)$. 


\section{Optimization OVER MULTI-OBJECTIVE SAM- PLES}

In this section we consider optimization problems where we have keys $\mathcal{X}$, nonnegative functions $f \in F$ where $f: \mathcal{X}$ and we seek to maximize $M(\operatorname{sum}(f))$ over $f \in F$ :

$$
f=\arg \max _{g \in F} M(\operatorname{sum}(g)) .
$$

We will assume here that the function $M$ is smooth with bounded rate of change: $\left|M(v)-M\left(v^{\prime}\right)\right| / M(v) \leq c\left|v-v^{\prime}\right| / v$, so that when $v^{\prime} \approx v$ then $M\left(v^{\prime}\right) \approx M(v)$.

Optimization over the large set $\mathcal{X}$ can be costly or infeasible and we therefore aim to instead compute an approximate maximizer over a multi-objective sample $S$ of $\mathcal{X}$. We propose a framework that adaptively increases the sample size until the approximate optimization goal is satisfied with the desired statistical guarantee.

We work with a slightly more general formulation where we allow the keys to have importance weights $m_{x} \geq 0$ and consider the weighted sums $\operatorname{sum}(f ; \mathcal{X}, \boldsymbol{m})=\sum_{x \in \mathcal{X}} f_{x} m_{x}$. Note that for the purpose of defining the problem, we can without loss of generality "fold" the weights $m_{x}$ into the functions $f \in F$ to obtain the set $F^{\prime} \equiv m F$ which has uniform importance weights so that $f^{\prime} \in F^{\prime}$ is defined from $f \in F$ using $\forall x f_{x}^{\prime}=f_{x} m_{x}$. When estimating from a sample, however, keys get re-weighted and therefore it is useful to separate out $F$, which may have a particular structure we need to preserve, and the importance weights $\boldsymbol{m}$.

Two example problem domains of such optimizations are clustering (where $\mathcal{X}$ are points, each $f \in F$ corresponds to a set of centers, $f_{x}$ depend on the distance from $x$ to the centers, and $\operatorname{sum}(f)$ is the cost of clustering with $f$ ) and empirical risk minimization (where $\mathcal{X}$ are examples and $\operatorname{sum}(f)$ is the loss of model $f)$. In these settings we seek to minimize $(M(v)=-v)$ or $\operatorname{maximize}(M(v)=v) \operatorname{sum}(f)$.

We present a meta-algorithm for approximate optimization that uses the following:

- Upper bounds $\pi_{x} \geq p_{x}^{(m F, 1)}$ on the base multi-objective pps probabilities. We would like $h=\sum_{x} \pi_{x}$ to be not much larger than $\sum p_{x}^{(F, 1)}$. (Here we denote by $m F$ the importance weights $\boldsymbol{m}$ folded into $F$ ).

- Algorithm $\mathcal{A}$ that for input $S \subset \mathcal{X}$ and positive weights $a_{x}$ for $x \in S$ returns $f \in F$ that (approximately) optimizes $M(\operatorname{sum}(f ; S, \boldsymbol{a}))$. By approximate optimum, we allow well-concentrated relative error with respect to the optimum $\max _{g \in F} M(\operatorname{sum}(g ; S, \boldsymbol{a}))$ or with respect to the optimum $\max _{g \in G} M(\operatorname{sum}(g ; S, \boldsymbol{a}))$ on a more restricted set $G \subset F$.

We apply this algorithm to samples $S$ obtained with probabilities $p_{x}=\min \left\{1, k \pi_{x}\right\}$. The keys $x \in S$ have importance weights $a_{x}=m_{x} / p_{x}$. Note that $\operatorname{sum}(g ; S, a)=$ $\sum_{x \in S} m_{x} g_{x} / p_{x}$, is the estimate of $\operatorname{sum}(g ; \mathcal{X}, \boldsymbol{m})$ we obtain from $S$.

Optimization over the sample requires that an (approximate) maximizer $f$ that meets our quality guarantees over the sample distribution is an approximate maximizer of (11). Intuitively, we would need that at least one approximate maximizer $f$ of (11) is approximated well by the sample $M(\operatorname{sum}(f ; S)) \approx$ $M(\operatorname{sum}(f ; \mathcal{X}))$ and that all $f$ that are far from being approximate maximizers are not approximate maximizers over the sample.
An ForAll sample is sufficient but pessimistic. Moreover, meeting ForAll typically necessitates worst-case non-adaptive bounds on sample size. An ForEach sample, obtained with $k \geq \epsilon^{-2}$, is not sufficient in and off itself, but a key observation is that maximization over a ForEach sample can only err (within our ForEach statistical guarantees) by over-estimating the maximum, that is, returning $f$ such that $M(\operatorname{sum}(f ; S, \boldsymbol{a})) \gg$ $M(\operatorname{sum}(f ; \mathcal{X}, \boldsymbol{m})$. Therefore, if

$$
\operatorname{sum}(f ; \mathcal{X}, \boldsymbol{m}) \geq(1-\epsilon) \operatorname{sum}(f ; S, \boldsymbol{a})
$$

we can certify, within the statistical guarantees provided by $\mathcal{A}$ and ForEach), that the sample maximizer $f$ is an approximate maximizer of 11. Otherwise, we obtain a lower and approximate upper bounds

$$
[M(\operatorname{sum}(f ; \mathcal{X}, \boldsymbol{m})),(1+\epsilon) M(\operatorname{sum}(f ; S, \boldsymbol{a}))]
$$

on the optimum. Finally, this certification can be done by exact computation of $\operatorname{sum}(f ; \mathcal{X}, \boldsymbol{m})$, but it can be performed much more efficiently with statistical guarantees using independent "validation" samples from the same distribution.

Algorithm 3 exploits this property to perform approximate optimization with an adaptive sample size. The algorithm starts with an ForEach sample. It iterates approximate optimization over the sample, testing (12), and doubling the sample size parameter $k$, until the condition (12) holds. Note that since the sample size is doubled, the ForEach guarantees tighten with iterations, thus, from concentration we get confidence for test results over the iterations. The algorithm uses the smallest sample size where probabilities are of the form $\min \left\{1, k \pi_{x}\right\}$. Note (see example in the previous section) that the optimization might be supported by a much smaller sample of a different form. An interesting open question is whether we can devise an algorithm that increases sampling probabilities in a more targeted way and can perform the approximate optimization using a smaller sample size.

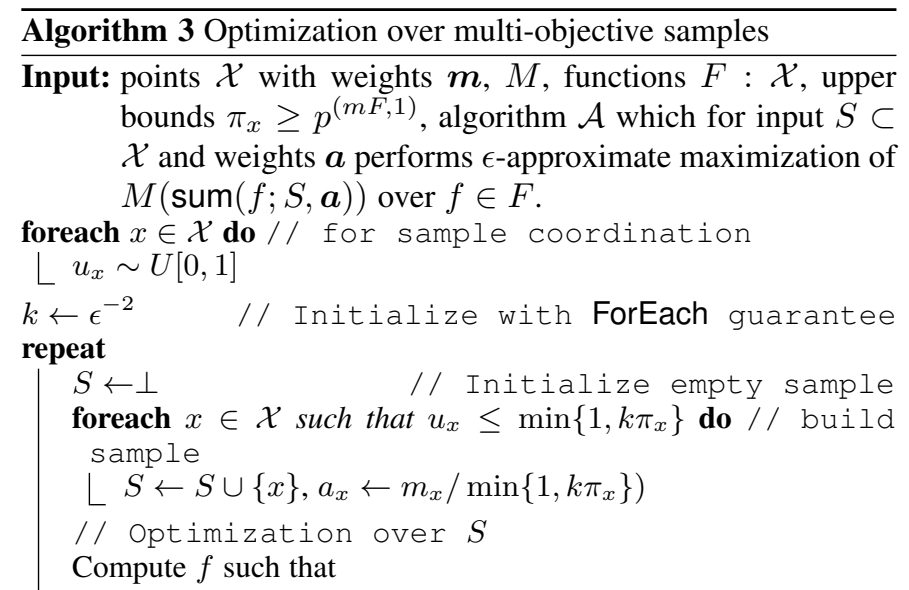

$$
M(\operatorname{sum}(f ; S, \boldsymbol{a})) \geq(1-\epsilon) \max _{g \in F} M(\operatorname{sum}(g ; S, \boldsymbol{a}))
$$

$k \leftarrow 2 k$ // Double the sample size until $M(\operatorname{sum}(f ; \mathcal{X})) \geq(1-\epsilon) M(\operatorname{sum}(f ; S))$ approx using a validation sample

return $f$

\section{Conclusion}

Multi-objectives samples had been studied and applied for nearly five decades. We present a unified review and extended analysis 
of multi-objective sampling schemes, geared towards efficient computation over very large data sets. We lay some foundations for further exploration and additional applications.

A natural extension is the design of efficient multi-objective sampling schemes for unaggregated data [19], [2] presented in a streamed or distributed form. The data here consists of data elements that are key value pairs, where multiple elements can share the same key $x$, and the weight $w_{x}$ is the sum of the values of elements with key $x$. We are interested again in summaries that support queries of the form $\operatorname{sum}(f ; H)$, where $f_{x}=f\left(w_{x}\right)$ for some function $f \in F$. To sample unaggregated data, we can first aggregate it and then apply sampling schemes designed for aggregated data. Aggregation, however, of streamed or distributed data, requires state (memory or communication) of size proportional to the number of unique keys. This number can be large, so instead, we aim for efficient sampling without aggregation, using state of size proportional to the sample size. We recently proposed such a sampling framework for capping statistics [9], which also can be used to for all statistics in their span.

\section{REFERENCES}

[1] P. K. Agarwal, S. Har-Peled, and K. R. Varadarajan. Geometric approximation via coresets. In Combinatorial and computational geometry, MSRI. University Press, 2005

[2] N. Alon, Y. Matias, and M. Szegedy. The space complexity of approximating the frequency moments. J. Comput. System Sci., 58:137-147, 1999.

[3] P. Boldi, M. Rosa, and S. Vigna. HyperANF: Approximating the neighbourhood function of very large graphs on a budget. In $W W W$, 2011.

[4] K. R. W. Brewer, L. J. Early, and S. F. Joyce. Selecting several samples from a single population. Australian Journal of Statistics, 14(3):231-239, 1972.

[5] M. T. Chao. A general purpose unequal probability sampling plan. Biometrika, 69(3):653-656, 1982.

[6] S. Chechik, E. Cohen, and H. Kaplan. Average distance queries through weighted samples in graphs and metric spaces: High scalability with tight statistical guarantees. In RANDOM. ACM, 2015.

[7] E. Cohen. Size-estimation framework with applications to transitive closure and reachability. J. Comput. System Sci., 55:441-453, 1997.

[8] E. Cohen. All-distances sketches, revisited: HIP estimators for massive graphs analysis. TKDE, 2015.

[9] E. Cohen. Stream sampling for frequency cap statistics. In KDD. ACM, 2015. full version: http://arxiv.org/abs/1502.05955.

[10] E. Cohen, S. Chechik, and H. Kaplan. Clustering over multi-objective samples: The one2all sample. CoRR, abs/1706.03607, 2017.

[11] E. Cohen, D. Delling, T. Pajor, and R. F. Werneck. Sketch-based influence maximization and computation: Scaling up with guarantees. In CIKM. ACM, 2014

[12] E. Cohen, N. Duffield, C. Lund, M. Thorup, and H. Kaplan. Efficient stream sampling for variance-optimal estimation of subset sums. SIAM J. Comput., 40(5), 2011

[13] E. Cohen, N. Grossuag, and H. Kaplan. Processing Top-k Queries from Samples. In Proceedings of the 2006 ACM conference on Emerging network experiment and technology (CoNext). ACM, 2006.

[14] E. Cohen and H. Kaplan. Summarizing data using bottom-k sketches. In ACM PODC, 2007.

[15] E. Cohen and H. Kaplan. Tighter estimation using bottom-k sketches. In Proceedings of the 34th VLDB Conference, 2008.

[16] E. Cohen, H. Kaplan, and S. Sen. Coordinated weighted sampling for estimating aggregates over multiple weight assignments. $V L D B, 2(1-2)$, 2009. full: http://arxiv.org/abs/0906.4560.

[17] N. Duffield, M. Thorup, and C. Lund. Priority sampling for estimating arbitrary subset sums. J. Assoc. Comput. Mach., 54(6), 2007.

[18] W. Feller. An introduction to probability theory and its applications, volume 2. John Wiley \& Sons, New York, 1971.

[19] P. Flajolet and G. N. Martin. Probabilistic counting algorithms for data base applications. J. Comput. System Sci., 31:182-209, 1985.

[20] R. Gandhi, S. Khuller, S. Parthasarathy, and A. Srinivasan. Dependent rounding and its applications to approximation algorithms. J. Assoc. Comput. Mach., 53(3):324-360, 2006.
[21] M. H. Hansen and W. N. Hurwitz. On the theory of sampling from finite populations. Ann. Math. Statist., 14(4), 1943.

[22] D. G. Horvitz and D. J. Thompson. A generalization of sampling without replacement from a finite universe. Journal of the American Statistical Association, 47(260):663-685, 1952.

[23] L. Kish and A. Scott. Retaining units after changing strata and probabilities. Journal of the American Statistical Association, 66(335):pp. 461-470, 1971.

[24] E. Ohlsson. Sequential poisson sampling. J. Official Statistics, 14(2):149-162, 1998.

[25] E. Ohlsson. Coordination of pps samples over time. In The 2nd International Conference on Establishment Surveys, pages 255-264. American Statistical Association, 2000.

[26] C. R. Palmer, P. B. Gibbons, and C. Faloutsos. ANF: A fast and scalable tool for data mining in massive graphs. In $K D D, 2002$.

[27] B. Rosén. Asymptotic theory for successive sampling with varying probabilities without replacement, I. The Annals of Mathematical Statistics, 43(2):373-397, 1972

[28] B. Rosén. Asymptotic theory for order sampling. J. Statistical Planning and Inference, 62(2):135-158, 1997.

[29] P. J. Saavedra. Fixed sample size pps approximations with a permanent random number. In Proc. of the Section on Survey Research Methods, pages 697-700, Alexandria, VA, 1995. American Statistical Association.

[30] C-E. Särndal, B. Swensson, and J. Wretman. Model Assisted Survey Sampling. Springer, 1992.

[31] M. Szegedy. The DLT priority sampling is essentially optimal. In Proc. 38th Annual ACM Symposium on Theory of Computing. ACM, 2006.

[32] Y. Tillé. Sampling Algorithms. Springer-Verlag, New York, 2006.

\section{APPENDIX}

Theorem A.1. Consider ppswor sampling with respect to weights $f_{x}$ and the estimator (2) computed using (3). Then for any $g \geq 0$ and segment $H, C V[\widehat{\operatorname{sum}}(g ; H)] \leq \sqrt{\frac{\rho(f, g)}{q^{(g)}(H)(k-1)}}$.

Proof. We adapt a proof technique in [9] (which builds on [7], [8]). To simplify notation, we use $W=\operatorname{sum}(f, \mathcal{X})=\sum_{x \in \mathcal{X}} f_{x}$ for the total $f$-weight of the population.

We first consider the variance of the inverse probability estimate for a key $x$ with weight $g_{x}$, conditioned on the threshold $\tau$. We use the notation $\hat{g}_{x}^{(\tau)}$ for the estimate that is $g_{x} / \operatorname{Pr}[f$-seed $(x)<\tau]$ when $f$-seed $(x)<\tau$ and 0 otherwise. Using $p=\operatorname{Pr}[f$-seed $(x)<\tau]=1-e^{-f_{x} \tau}$, we have

$$
\begin{aligned}
\operatorname{var}\left[\hat{g}_{x}^{(\tau)}\right] & =\frac{1-p}{p} g_{x}^{2}=g_{x}^{2} \frac{e^{-\tau f_{x}}}{1-e^{-\tau f_{x}}} \\
& \leq \frac{g_{x}^{2}}{f_{x} \tau} \leq \max _{y} \frac{g_{y}}{f_{y}} \frac{g_{x}}{\tau},
\end{aligned}
$$

using the relation $e^{-z} /\left(1-e^{-z}\right) \leq 1 / z$.

We now consider the variance of the estimator $\hat{g}_{x}^{(\tau)}$ when $\tau$ is the $k$ th smallest seed value $\tau^{\prime}$ in $\mathcal{X} \backslash x$. We denote by $B_{x}$ the distribution of $\tau^{\prime}$. We will bound the variance of the estimate using the relation

$$
\operatorname{var}\left[\hat{g}_{x}\right]=\mathrm{E}_{\tau^{\prime} \sim B_{x}} \operatorname{var}\left[\hat{g}_{x}^{\left(\tau^{\prime}\right)}\right] .
$$

The distribution of $\tau^{\prime}$ is the $k$ th smallest of independent exponential random variables with parameters $f_{y}$ for $y \in \mathcal{X} \backslash x$. From properties of the exponential distribution, the minimum seed is exponentially distributed with parameter $W-f_{x}$, the difference between the minimum and second smallest is exponentially distributed with parameter $W-f_{x}-w_{1}$, where $w_{1}$ is the weight $f_{y}$ of the key $y$ with minimum seed, and so on. Therefore, the distribution on $\tau^{\prime}$ conditioned on the ordered set of smallest-seed keys is a sum of $k$ exponential random variables with parameters at most $W$. The distribution $B_{x}$ is a convex combination of such distributions. We use the notation $s_{W, k}$ for the density function of the Erlang distribution $\operatorname{Erlang}(W, k)$, which is a sum of $k$ 
independent exponential distribution with parameter $W$. What we obtained is that the distribution $B_{x}$ (for any $x$ ) is dominated by $\operatorname{Erlang}(W, k)$.

Since our bound on the conditioned variance $\operatorname{var}\left[\hat{g}_{x}^{\left(\tau^{\prime}\right)}\right]$ is nonincreasing with $\tau^{\prime}$, domination implies that

$$
\mathrm{E}_{\tau^{\prime} \sim B_{x}} \operatorname{var}\left[\hat{g}_{x}^{\left(\tau^{\prime}\right)} \leq \mathrm{E}_{\tau^{\prime} \sim \operatorname{Erlang}(W, k)} \overline{\operatorname{var}}\left[\hat{g}_{x}^{\left(\tau^{\prime}\right)}\right],\right.
$$

where $\overline{\mathrm{var}}$ is our upper bound (13). We now use the Erlang density function [18]

$$
s_{W, k}(z)=\frac{W^{k} z^{k-1}}{(k-1) !} e^{-W z}
$$

and the relation $\int_{0}^{\infty} z^{a} e^{-b z} d z=a ! / b^{a+1}$ to bound the variance:

$$
\begin{aligned}
\operatorname{var}\left[\hat{g}_{x}\right] & \leq \int_{0}^{\infty} s_{W, k}(z) \overline{\operatorname{var}}\left[\hat{g}_{x}^{(z)}\right] d z \\
& \leq \int_{0}^{\infty} \frac{W^{k} z^{k-1}}{(k-1) !} e^{-W z} \frac{g_{x}}{z} \max _{y} \frac{g_{y}}{f_{y}} d z \\
& \leq \max _{y} \frac{g_{y}}{f_{y}} g_{x} \frac{W^{k}}{(k-1) !} \int_{0}^{\infty} z^{k-2} e^{-W z} d z \\
& =\max _{y} \frac{g_{y}}{f_{y}} \frac{g_{x} W}{k-1} .
\end{aligned}
$$

By definition, $\widehat{\operatorname{sum}}(g ; H)=\sum_{x \in H} \hat{g}_{x}$. Since covariances between different keys are zero [15], $\operatorname{var}[\widehat{\operatorname{sum}}(g ; H)]=$ $\sum_{x \in H} \operatorname{var}\left[\hat{g}_{x}\right] \leq \max _{y} \frac{g_{y}}{f_{y}} \frac{\operatorname{sum}(g ; H) W}{k-1}$.

$$
\begin{aligned}
& \mathrm{CV}[\widehat{\operatorname{sum}}(g ; H)]^{2}=\frac{\operatorname{var}[\widehat{\operatorname{sum}}(g ; H)]}{\operatorname{sum}(g ; H)^{2}} \leq \max _{y} \frac{g_{y}}{f_{y}} \frac{\operatorname{sum}(g ; H) W}{(k-1) \operatorname{sum}(g ; H)^{2}} \\
& \leq \max _{y} \frac{g_{y}}{f_{y}} \frac{1}{k-1} \frac{W}{\operatorname{sum}(g ; H)} \\
& \leq \max _{y} \frac{g_{y}}{f_{y}} \frac{1}{k-1} \frac{\operatorname{sum}(g ; \mathcal{X})}{\operatorname{sum}(g ; H)} \frac{W}{\operatorname{sum}(g ; \mathcal{X})} \leq \frac{\rho}{q(k-1)} .
\end{aligned}
$$

